archives-ouvertes

\title{
Longitudinal soliton tunneling in optical fiber
}

T. Marest, F. Braud, M. Conforti, S. Wabnitz, A. Mussot, And Kudlinski

\section{To cite this version:}

T. Marest, F. Braud, M. Conforti, S. Wabnitz, A. Mussot, et al.. Longitudinal soliton tunneling in optical fiber. Optics Letters, Optical Society of America, 2017, 42 (12), pp.2350. 10.1364/OL.42.002350 . hal-02386225

\section{HAL Id: hal-02386225 \\ https://hal.archives-ouvertes.fr/hal-02386225}

Submitted on 29 Nov 2019

HAL is a multi-disciplinary open access archive for the deposit and dissemination of scientific research documents, whether they are published or not. The documents may come from teaching and research institutions in France or abroad, or from public or private research centers.
L'archive ouverte pluridisciplinaire HAL, est destinée au dépôt et à la diffusion de documents scientifiques de niveau recherche, publiés ou non, émanant des établissements d'enseignement et de recherche français ou étrangers, des laboratoires publics ou privés. 


\title{
Longitudinal soliton tunneling in optical fiber
}

\author{
T. Marest ${ }^{1}$, F. Braud ${ }^{1}$, M. Conforti ${ }^{1}$, S. Wabnitz ${ }^{2}$, A. Mussot ${ }^{1}$, and A. Kudlinski ${ }^{1, *}$ \\ ${ }^{1}$ Université Lille, CNRS, UMR 8523-PhLAM-Physique des Lasers Atomes et Molécules, F-59000 Lille, France \\ ${ }^{2}$ INO CNR and Dipartimento di Ingegneria dell'Informazione, Università di Brescia, Via Branze 38, Brescia, Italy \\ *Corresponding author: alexandre.kudlinski@univ-lille1.fr
}

Compiled April 21, 2017

\begin{abstract}
We report the observation of the longitudinal soliton tunneling effect in axially-varying optical fibers. A fundamental soliton, initially propagating in the anomalous dispersion region of a fiber, $c$ an $p$ ass $t$ hrough a normal dispersion barrier without being substantially affected. We perform experimental studies by means of spectral and temporal characterizations that show the evidence of longitudinal soliton tunneling process. Our results are well supported by numerical simulations using the generalized nonlinear Schrödinger equation.
\end{abstract}

OCIS codes: (190.4370) Nonlinear optics, fibers; (190.5530) Pulse propagation and temporal solitons.

http://dx.doi.org/10.1364/ao.XX.XXXXXX

A temporal optical soliton is a shape invariant localized pulse resulting from the balance between focusing Kerr nonlinearity and anomalous group velocity dispersion (GVD) [1]. Since the very first introduction of the word soliton in plasma physics, particular emphasis was given to the particle-like nature of these waves [2]. Solitons can collide elastically between each other [3] and can survive severe perturbations, like Raman effect [4], high-order dispersion [5] or the interaction with linear waves [6]. Studies of the scattering of a soliton approaching a potential have shown a dynamics similar to the quantum particle case [7] in terms of transmission or reflection by the potential barrier. In particular, a soliton has the ability to tunnel trough a potential in analogy with the quantum tunneling effect [8-10]. The similarity between optical tunneling in fibers and quantum tunneling has been pointed out, for example, in [11] in the spectral domain, where the potential barrier is mimicked by a sign change of GVD [12]. The spectral tunneling effect was also experimentally studied in microstructured fibers [13, 14]. In these works, the fiber was designed to have two closely-separated zero dispersion wavelengths (ZDWs), so that two different anomalous GVD regions are separated by a narrow normal GVD region, acting as a potential barrier. A soliton propagating in the first anomalous GVD region is red-shifting via self-induced Raman scattering until it reaches one ZDW, where the frequency shift stops [4]. This cancellation of the Raman self-frequency shift is accompanied by the emission of a phase-matched dispersive wave across the ZDW. When the dispersion profile is properly designed, the phase-matched dispersive wave will be emitted across the potential barrier in the second, long wavelength side anomalous GVD region, where solitons can exist. Therefore, there is a continuous flow of energy from the initial soliton to the emitted dispersive wave, which in turn may lead to the formation of a new soliton, resulting in an overall soliton tunnelling effect across the GVD barrier.

In the present work, we propose and study, both numerically and experimentally, a different kind of optical soliton tunneling which is, once again, analogous to the quantum tunneling effect. In this case, the potential barrier is a short normal GVD fiber segment, placed between two anomalous GVD fiber sections. The soliton propagation in such a system can be divided into three parts. In the initial stage, we generate a soliton propagating in a fiber with anomalous GVD. After some propagation distance, the soliton reaches the normal GVD barrier, where it cannot exist anymore as a soliton, and thus it excites a dispersive pulse. Finally, the pulse reaches the final anomalous GVD segment, where it reshapes once again into a soliton.

Let us begin our study with numerical simulations based on the generalized nonlinear Schrödinger equation (GNLSE) [15]:

$$
i \partial_{z} A+D\left(i \partial_{t}, z\right) A+\gamma\left(A \int R\left(t^{\prime}\right)\left|A\left(t-t^{\prime}\right)\right|^{2} d t^{\prime}\right)=0
$$

where the dispersion operator $D\left(i \partial_{t}\right)=\sum_{n \geq 2} \frac{\beta_{n}}{n !}\left(i \partial_{t}\right)^{n}$ takes into account the dispersion profile of the fiber up to $n=3$ and where $\gamma$ is the nonlinear parameter. $R(t)=\left(1-f_{R}\right) \delta(t)+$ $f_{R} h_{R}(t)$ includes both Kerr and Raman effects, where $h_{R}(t)$ correspond to the Raman response function $\left(f_{R}=0.18\right)$. $D\left(i \partial_{t}\right)$ is expanded around $\omega_{p}$, the pump carrier frequency, and $t$ is the retarded time in the frame traveling at the group velocity $V_{g}=V_{g}\left(\omega_{p}\right)=\beta_{1}^{-1}$ of the pump pulses. We have verified that the self-steepening term does not play any important role. We consider a concatenation of three dispersion shifted fiber (DSF) segments with different values of ZDW. The present study is made with a view to experiments, thus we choose all parameters in the range of available fibers. Accordingly, the first section has a ZDW equal to $1385 \mathrm{~nm}$ and a length of $50 \mathrm{~m}$. Next, the ZDW of the second fiber (acting as the barrier at the origin of the tunneling effect) goes up to $1700 \mathrm{~nm}$ for $3 \mathrm{~m}$. Finally, the ZDW of the third $50 \mathrm{~m}$ long fiber segment drops down to 1490 $\mathrm{nm}$. In this last section, the ZDW was set higher than the ZDW of the first section, in order to excite a low order soliton after the normal GVD barrier, when taking into account the soliton Raman self-frequency shift (SSFS) of the incident soliton in the 
first fiber segment. The pump is set to our available laser i.e. a Gaussian pulse centered around $1485 \mathrm{~nm}$ and with a full width at half-maximum (FWHM) duration of $220 \mathrm{fs}$. At this wavelength, the nonlinear parameter $\gamma$ is about $5\left(W . \mathrm{km}^{-1}\right.$ and the third order dispersion $\beta_{3}$ is equal to $5 \mathrm{ps}^{3} / \mathrm{m}$ for all sections.

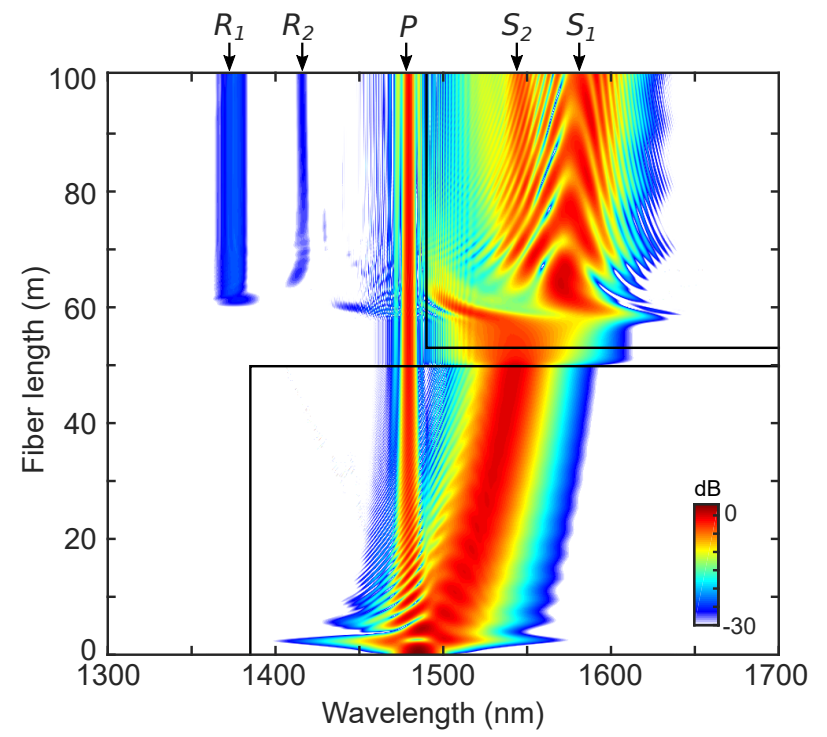

Fig. 1. Numerical simulation of experiments demonstrating the longitudinal soliton tunneling effect. Simulated spectrum against the fiber length. The black line corresponds to the ZDW and $R_{1}, R_{2}, P, S_{1}, S_{2}$ respectively stand for the different components of the output spectrum: radiation 1 and 2, pump residue, soliton 1 and 2 .

Figure 1 shows the simulated spectral dynamics along the propagation coordinate in the fiber for an input pulse centered around $1485 \mathrm{~nm}$ (launched in anomalous GVD region), and a peak power $P_{\text {peak }}$ of $240 \mathrm{~W}$. At the initial stage of propagation, we observe the generation of a fundamental soliton and pump residues (labeled $P$ in Fig. 1). Undergoing SSFS, the ejected soliton reaches $1542 \mathrm{~nm}$ at the end of the first fiber section, at $z=50 \mathrm{~m}$. In the second fiber section, where the GVD is normal, the pulse experiences strong dispersion and its peak power decreases, so that the Raman frequency shift rapidly stops. Afterwards, the pulse reaches the last fiber section at $z=53 \mathrm{~m}$, where again propagation occurs in the anomalous GVD region. From about $60 \mathrm{~m}$, we can identify the formation of two solitary pulses (labeled $S_{1}$ and $S_{2}$ ), which reach $1547 \mathrm{~nm}$ and $1590 \mathrm{~nm}$ at $100 \mathrm{~m}$, respecively. Simultaneously, two small radiative waves are generated at $1375 \mathrm{~nm}$ and $1416 \mathrm{~nm}$, which is consistent with the known picture of the generation of dispersive waves from solitons. Indeed, it can be verified that $S_{1}$ and $R_{1}$ on one hand, and $S_{2}$ and $R_{2}$ on the other hand both satisfy the phase matching relation linking dispersive waves to solitons [5]. So at this point, it appears that the initial soliton has been able to tunnel through the normal GVD barrier, giving rise to two solitons after the potential barrier.

To get further insight into this process, we studied its dynamics in the time domain. Figure 2(a) shows the temporal profile along the propagation distance in the fiber, whereas Fig. 2(b) illustrates the temporal profile at the end of the initial 50 m long anomalous GVD section (blue curve), at the end of the second normal GDV segment (green curve), and at the end of final anomalous GVD section (red curve), respectively. At the
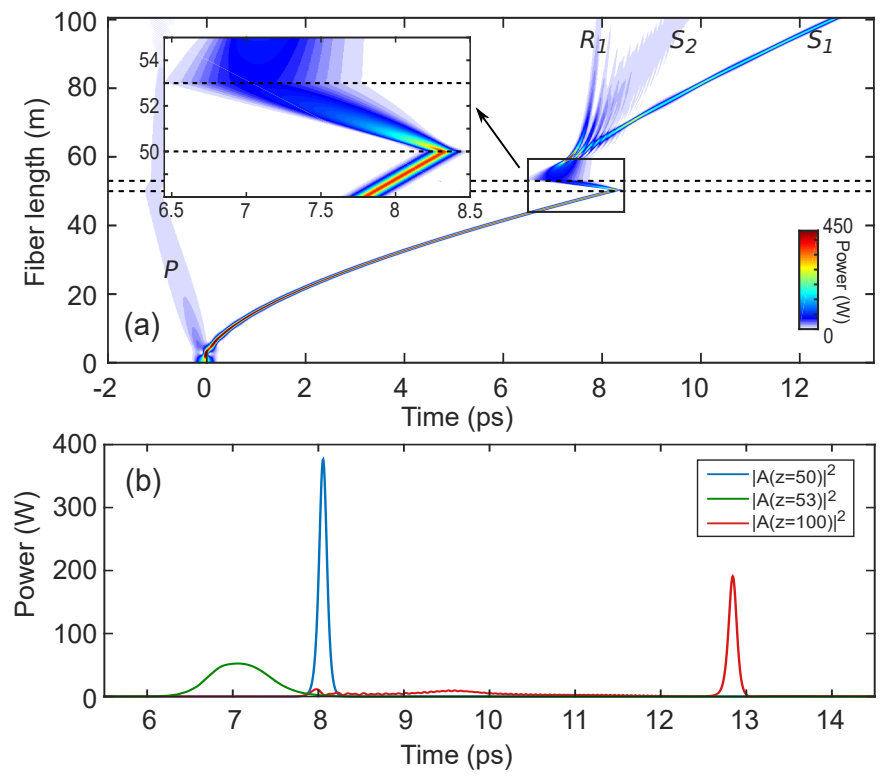

Fig. 2. (a) Simulated temporal profile against the fiber length. The dashed horizontal lines mark the normal GVD tunnel. (b) Temporal profile respectively at $z=50 \mathrm{~m}$ (blue), $z=53 \mathrm{~m}$ (green) and at the fiber output $z=100 \mathrm{~m}$ (red).

initial stage, the soliton rapidly separates from the pump $P$ and strongly decelerates due to SSFS, as observed in the spectral domain in Fig. 1. The soliton reaches the normal GVD fiber section input at $50 \mathrm{~m}$ (at around $8 \mathrm{ps}$ in the plot), with a FWHM duration of $118 \mathrm{fs}$ [blue curve in Fig. 2(b)]. Inside the second fiber section, the pulse strongly broadens temporally due to normal GVD. At the end of this segment (at $53 \mathrm{~m}$ ), the pulse loses its hyperbolic secant shape and it acquires a Gaussian profile, with a FWHM duration of $784 \mathrm{fs}$ [green curve in Fig. 2(b)], which is more than 6 times the FWHM duration at the input of the normal GVD fiber $(50 \mathrm{~m})$. Afterwards, the pulse enters the final anomalous dispersion fiber section, where it temporally recompresses and reshapes into a fundamental soliton. By comparing with the soliton entering the tunnel, the peak power of this soliton $S_{1}$ is reduced by a factor of about 2 , while its duration is about the same. A second weak pulse $S_{2}$ can be also observed around 9.5 ps at the end of the third fiber. Performing a simulation over a much longer distance of $200 \mathrm{~m}$, we noticed that the $S_{2}$ pulse remains stable as a second (weak) soliton. A radiation peak corresponding to $R_{1}$ is observed from $60 \mathrm{~m}$ in Fig. 2(a). The second radiation peak, $R_{2}$ is too weak to be seen on this plot, although we verified its presence by adapting the colorscale (not shown here). These results provide a theoretical evidence of the process of longitudinal soliton tunnelling, in which a soliton can tunnel through a potential barrier made of a short normal GDV fiber section.

We now focus our study on the impact of the normal GVD fiber length on the tunnelling process. To do that, we analyze the shape of the output field from the third fiber, while keeping the length of the last fiber section constant and equal to 50 $\mathrm{m}$ and progressively increasing the length of the normal GVD section length. To perform this analysis, we numerically solved the Zakharov-Shabat (ZS) direct scattering problem [16] with the Fourier collocation method [17], with as initial condition the output pulse from the third fiber section. Neglecting higherorder dispersive effects and Raman effect in Eq. 1, the GNLSE 


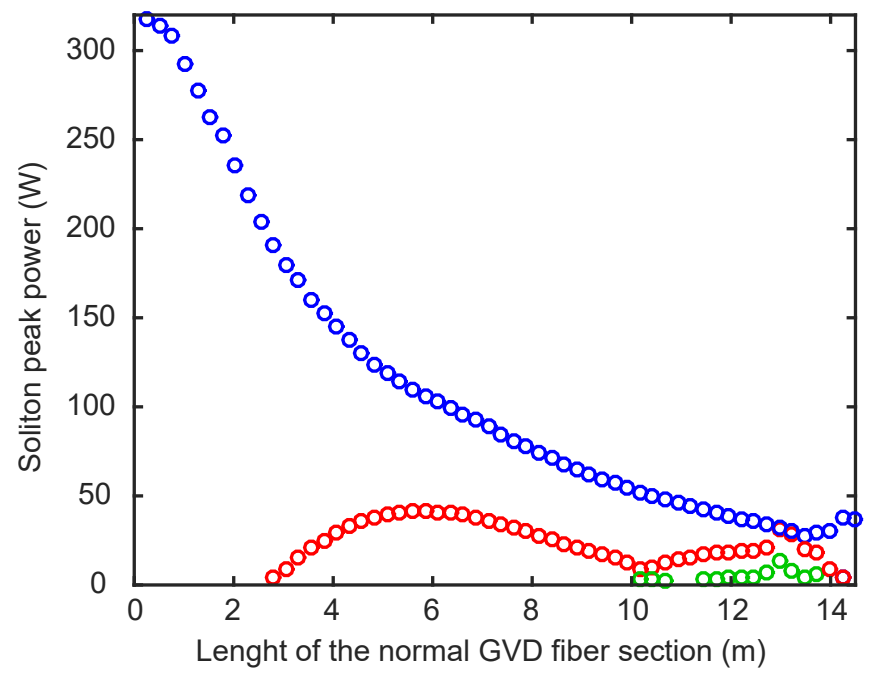

Fig. 3. Soliton power at the fiber output against the normal GVD section length, obtained by solving the Zakharov-Shabat direct scattering problem. One color corresponds to one soliton.

reduces to the integrable NLSE where inverse scattering technique applies. Additional details on the method can be found in [18]. The discrete eigenvalues of the ZS problem are directly related to the soliton peak power and velocity. Here, we consider the simulated output field, in which the pump residue has been filtered out, obtained for different lengths of the normal GVD segment. The corresponding results are shown in Fig. 3 where we have plotted the output soliton peak power against the normal GVD barrier length. The different colors stand for the different discrete eigenvalues of the ZS problem, i.e. for different solitons. First, we may note that obtaining discrete eigenvalues of the ZS problem demonstrates that the pulses coming out the third fiber are in fact fundamental solitons. Second, we may note that the peak power of the main output soliton decreases with increasing length of the normal GVD fiber segment (as a reminder, the peak power of the soliton entering this section is $368 \mathrm{~W}$ ). This could be intuitively anticipated, since increasing the length of the normal GVD fiber is equivalent to an increase of the size of the potential well. When the normal GVD fiber length reaches $3 \mathrm{~m}$, a second soliton is generated (red markers). Figure 3 also shows that a third soliton is generated after $10 \mathrm{~m}$ (green markers). As the number of generated solitons keeps increasing, the peak power of the main soliton (blue markers) keeps decreasing. In the configuration that we numerically investigated in Figs. 1 and 2, i.e. a length of the normal GVD fiber of $3 \mathrm{~m}$, the plot of Fig. 3 indicates that one should asymptotically observe (in the absence of Raman effect and higher-order GVD) a main soliton $S_{1}$ with a peak power of $180 \mathrm{~W}$ and a very small soliton $S_{2}$ of peak power $9 \mathrm{~W}$, in good quantitative agreement with our numerical results.

In order to study the soliton tunneling effect experimentally, we constructed a composite fiber by splicing three dispersion shifted fibers with length and ZDW corresponding to the parameters of the previous numerical simulations. In particular, the length of the intermediate normal GVD fiber was set to 3 $\mathrm{m}$. We used an optical parametric oscillator (OPO) pumped by a Ti:Sa laser to generate the initial soliton. Our source delivers Gaussian pulses of 220 fs FWHM duration, which are tuned to

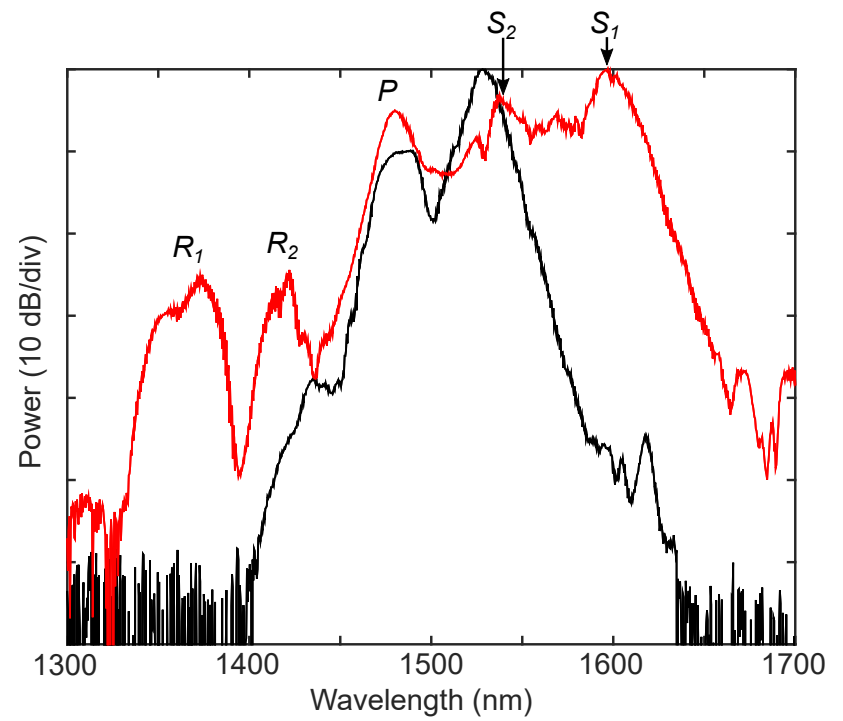

Fig. 4. Experimental spectrum measurement at $z=50 \mathrm{~m}$ (black curve) and at the fiber output $z=100 \mathrm{~m}$ (red curve).

$\lambda_{\text {pump }}=1485 \mathrm{~nm}$. The pulse power was controlled by means of a half wave plate and polarizers, and was set to $P_{\text {peak }}=240$ W. We used an optical spectrum analyzer to perform spectral measurements at the fiber output. The result is shown in Fig. 4, where we compare the experimental spectrum at the composite fiber output ( $z=100 \mathrm{~m}$, red line) with the spectrum measured at the input of the normal GVD section $(z=50 \mathrm{~m}$, black line) after a cutback. In Fig. 4 we can clearly identify the incident soliton entering the normal GVD section at $1528 \mathrm{~nm}$ (black line), along with the two output solitons around $1536 \mathrm{~nm}\left(S_{2}\right)$ and 1597 $\mathrm{nm}\left(S_{1}\right)$ on the red curve. In the output spectrum, we also see the two dispersive waves $\left(R_{1}\right.$ around $1365 \mathrm{~nm}$ and $R_{2}$ around $1420 \mathrm{~nm}$ ) generated from the two solitons $S_{1}$ and $S_{2}$, in excellent agreement with the previous numerical simulations.

In addition to these spectral measurements, we performed autocorrelation measurements after successive cutbacks of the fiber. This allowed us to follow the evolution of the main soliton temporal duration versus fiber length. To do that, the main soliton was spectrally filtered and sent to a second harmonic generation autocorrelator. Figures 5(a), (b) and (c) show three examples of experimental autocorrelation traces (red dashed lines) recorded at $20 \mathrm{~m}$ (i.e. in the initial anomalous GVD fiber), at $53 \mathrm{~m}$ (i.e. at the end of the normal GVD section) and at $93 \mathrm{~m}$ (in the final anomalous GVD fiber), respectively. In both anomalous GVD fiber segments, auto-correlations are well fitted by square hyperbolic secant functions (black lines), while in the normal GVD fiber, the optimal fitting function is Gaussian (black line). These measurements as well as additional ones are shown in Fig. 5(d) as red full circles, together with the simulation result (black solid line), which turns out to be in excellent agreement. One can see that the pulse quickly spreads in time when it enters in the normal GVD fiber "potential barrier" at $50 \mathrm{~m}$. The maximum FWHM duration (690 fs) is observed when the pulse reaches the end of this fiber segment $(z=53 \mathrm{~m})$. As soon as the pulse enters the final anomalous GVD fiber section, it recompresses in time until it reaches $60 \mathrm{~m}$, where the duration progressively stabilizes to around $125 \mathrm{fs}$, in excellent agreement with the simulation results. Its wavelength is $1590 \mathrm{~nm}$, again in full accordance with numerical predictions. These autocorrelation measurements 

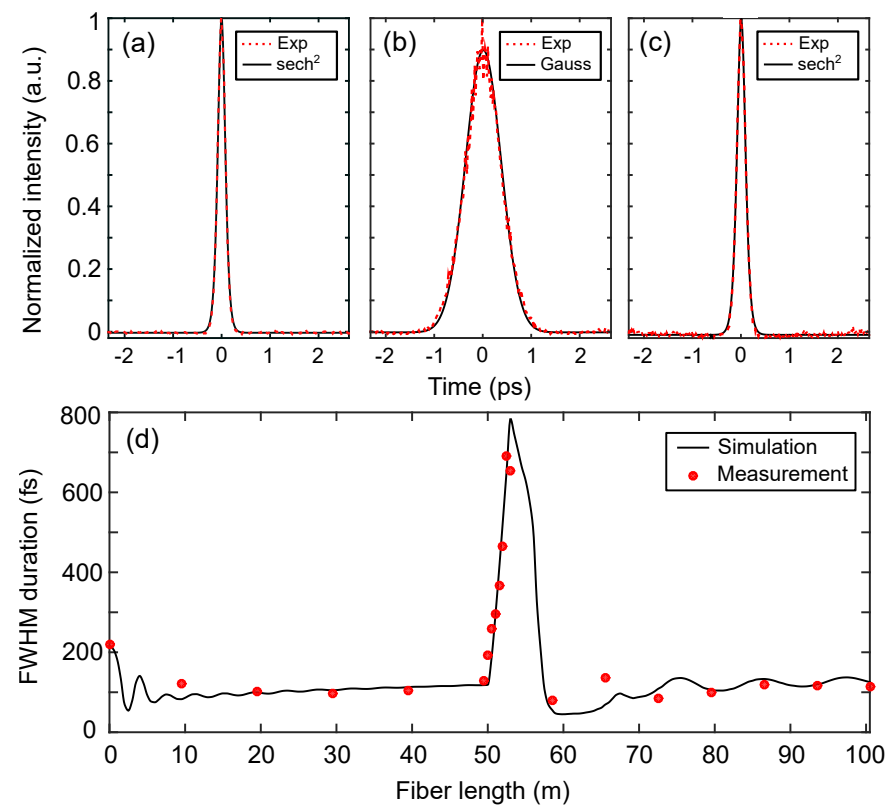

Fig. 5. Experimental auto-correlation trace evolution in red dotted curves at (a) $20 \mathrm{~m}$, (b) $53 \mathrm{~m}$, (c) $93 \mathrm{~m}$. The black lines correspond to the adjustment on a Gaussian pulse or hyperbolic secant, respectively. (d) Comparison between experimental measurement (red dots) and simulated FWHM duration evolution (black curve) against fiber length.

confirm the solitonic nature of the pulse before and after the normal GDV fiber section, and thus provide a clear experimental confirmation of the longitudinal soliton tunnelling effect.

To conclude, we observed for the first time to our knowledge, a new kind of optical soliton tunneling where a soliton can longitudinally pass through a normal GVD fiber section without experiencing substantial modifications of its properties. In analogy with the quantum tunneling effect, in which the transmission is set by the potential barrier strength, the length of the normal GVD fiber acting as the potential well has a strong influence on the efficiency of the process. Longitudinal soliton tunneling provides a fundamentally new example of optical invisibility (of the potential well barrier) which may find applications to the cloaking of information.

\section{ACKNOWLEDGEMENT}

This work has been partially supported by IRCICA, by the Agence Nationale de la Recherche through the TOPWAVE and NOAWE projects, the LABEX CEMPI (ANR-11-LABX-0007) and the Equipex Flux (ANR-11-EQPX-0017 projects), by the Ministry of Higher Education and Research, Hauts-de-France Regional Council and European Regional Development Fund (ERDF) through the CPER Photonics for Society P4S; by the Ministero dell' Istruzione, dell' Università e della Ricerca (MIUR) (PRIN 2015KEZNYM); and by the European Union's Horizon 2020 research and innovation programme under the Marie SkłodowskaCurie grant agreement No 691051.

\section{REFERENCES}

1. A. Hasegawa and M. Matsumoto, Optical solitons in fibers, Spinger (2003)

2. N. J Zabusky and M. D. Kruskal, Phys. Rev. Lett. 15, 240 (1965)
3. Y. Kivshar and B. Malomed, Rev. Mod. Phys. 45, 763 (1989).

4. D. V. Skryabin, F. Luan, J. C. Knight and P. St. J. Russell, Science 301, 1705 (2003).

5. N. Akhmediev and M. Karlsson, Phys. Rev. A 51, 2602 (1995).

6. S. F. Wang, A. Mussot, M. Conforti, X. L. Zeng, and A. Kudlinski, Opt. Lett. 40, 3320 (2015).

7. B. Gertjerenken, T.P. Billam, L. Khaykovich and C. Weiss, Pys. Rev. A 86, 033608 (2012).

8. C. H. Wang, T. M. Hong, R. K. Lee and D. W. Wang, Opt. Express 20, 22675 (2012).

9. S. Wang, H. Guo, D. Fan, X. Bai and X. Zeng, IEEE Photon. J. 5, 6100608 (2013).

10. D. Anderson, M. Lisak, B. Malomed and M. Quiroga-teixeiro, J. Opt. Soc. Am. B 11, 2380 (1994).

11. V. N. Serkin, V. A. Vysloukh and J. R. Taylor, Electron. Lett. 29, 12 (1993).

12. E. N. Tsoy and C. Martjin de Sterke, Phys. Rev. A 76, 043804 (2007).

13. F. Poletti, P. Horak and D. J. Richardson, IEEE Photon. Technol. Lett. 20, 1414 (2008).

14. H. Guo, S. Wang, X. Zeng and M. Bache, IEEE Photon. Technol. Lett. 25, 1928 (2013).

15. J. M. Dudley, G. Genty, and S. Coen, Rev. Mod. Phys. 78, 1135 (2006).

16. V. E. Zakharov and A. B. Shabat, Sov. Phys. J. Exp. Theor. Phys. 34, 62 (1972).

17. J. Yang, Nonlinear Waves in Integrable and Nonintegrable Systems, 1st ed. (SIAM, 2012).

18. F. Braud, M. Conforti, A. Cassez, and A. Kudlinski, Opt. Lett. 41, 1412 (2016). 


\section{INFORMATIONAL FIFTH PAGE}

\section{REFERENCES}

1. A. Hasegawa and M. Matsumoto, Optical solitons in fibers, Spinger (2003)

2. N. J Zabusky and M. D. Kruskal, Interaction of "Solitons" in a collisionless plasma and the recurrence of intial states, Phys. Rev. Lett. 15, 240 (1965)

3. Y. Kivshar and B. Malomed, Dynamics of solitons in nearly integrable systems, Rev. Mod. Phys. 45, 763 (1989).

4. D. V. Skryabin, F. Luan, J. C. Knight and P. St. J. Russell, Soliton SelfFrequency Shift Cancellation in Photonic Crystal Fibers, Science 301, 1705 (2003).

5. N. Akhmediev and M. Karlsson, Cherenkov radiation emitted by solitons in optical fibers, Phys. Rev. A 51, 2602 (1995).

6. S. F. Wang, A. Mussot, M. Conforti, X. L. Zeng, and A. Kudlinski, Bouncing of a dispersive wave in a solitonic cage, Opt. Lett. 40, 3320 (2015).

7. B. Gertjerenken, T.P. Billam, L. Khaykovich and C. Weiss, Scattering bright solitons: Quantum versus mean-field behavior, Phys. Rev. A 86, 033608 (2012).

8. C. H. Wang, T. M. Hong, R. K. Lee and D. W. Wang, Particle-wave duality in quantum tunneling of a bright soliton, Opt. Express 20, 22675 (2012).

9. S. Wang, H. Guo, D. Fan, X. Bai and X. Zeng, Analysis of Cascaded Soliton Spectral Tunneling Effect in Segmented Fibers With Engineered Dispersion, IEEE Photon. J. 5, 6100608 (2013).

10. D. Anderson, M. Lisak, B. Malomed and M. Quiroga-teixeiro, Tunneling of an optical soliton through a fiber junction, J. Opt. Soc. Am. B 11, 2380 (1994).

11. V. N. Serkin, V. A. Vysloukh and J. R. Taylor, Soliton spectral tunneling effect, Electron. Lett. 29, 12 (1993).

12. E. N. Tsoy and C. Martjin de Sterke, Theoretical analysis of the selffrequency shift near zero-dispersion points: Soliton spectral tunneling, Phys. Rev. A 76, 043804 (2007).

13. F. Poletti, P. Horak and D. J. Richardson, Soliton spectral tunneling in dispersion controlled holey fibers, IEEE Photon. Technol. Lett. 20, 1414 (2008).

14. H. Guo, S. Wang, X. Zeng and M. Bache, Understanding Soliton Spectral Tunneling as a Spectral Coupling Effect, IEEE Photon. Technol. Lett. 25, 1928 (2013).

15. J. M. Dudley, G. Genty, and S. Coen, Supercontinuum generation in photonic crystal fiber, Rev. Mod. Phys. 78,1135 (2006).

16. V. E. Zakharov and A. B. Shabat, Exact theory of two-dimensional selffocusing and one-dimensional self-modulation of waves in nonlinear media, Sov. Phys. J. Exp. Theor. Phys. 34, 62 (1972).

17. J. Yang, Nonlinear Waves in Integrable and Nonintegrable Systems, 1st ed. (SIAM, 2012).

18. F. Braud, M. Conforti, A. Cassez, and A. Kudlinski, Solitonization of a dispersive wave, Opt. Lett. 41, 1412 (2016). 